UCID--19561

DE83 000442

PARTICLE AND POWER PROFILE CALCULATIONS

FOR THE ATA BEAM DUMP AND

EMITTANCE BOX*

D. R. SLAUGHTER

August 19, 1982

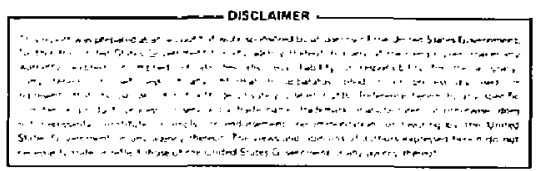

* Lawrence Livermore National Laboratory is operated by the University of California for the U. S. Department of Energy under Contract No. W-7405-Eng-48.

This work is performed by LLNL for the U. S. Department of Defense ulider DARPA (DOD) ARPA Order No. 4395 A:1, monitored by NSHC undi: document number N60921-8i-WR-W0056. 
Abstract

Electron-photon transport calculations have been carried out for the $50 \mathrm{MeV}$ electron beam generated by ATA when it is incident on a carbon beam dump. Energy spectra are calculated at various points within the dump for the purpose of determining the power density profile. In addition, energy spectra of scattered electrons transported through small holes in the dump are calculated for the purpose of determining the extent to which they would interfere with a beam-emittance and beam-energy diagnostic based on the extraction of small beamlets through penetrations in the dump. 
Introduction

The ATA ${ }^{(1)}$ at LLNL generates an electron beam of up to $10^{4}$ amps at 50 $\mathrm{MeV}$ in a pulse whose duration is up to $70 \mathrm{~ns}$. The pulses may be delivered in bursts at rates up to $10^{3}$ pulses per second and the average pulse rate over Iong times may be as high at 5 pulses per second. Since the beam spot may be as small as $1 \mathrm{~cm}$ in radius, the power density in the beam is large and the corresponding power density due to energy deposition in the beam dump is also large. Most naturally occurring elements would be melted by a rapid burst of ten ATA pulses (2) incident on a solid dump.

A series of electron-photon transport calculations has been carried out using the monte carlo code SANOYL ${ }^{(3)}$. Two problems have been explored in some detail. First, energy spectra and intensities have been caiculated in many regions of a solid carbon beam dump and these results were used to calculate power density profiles due to energy deposition by the electron beam and by its secondary photons. Carbon was chosen for study since it is the material which will withstand the greatest beam current density without. melting (2) and because it results in the lawest secondary neutron generation. (2) Second, useful beam diagnostic information could be obtained from time resolved energy and current measurements on beamlets extracted through an array of small holes in the dump. In order to assess the viability of such a diagnostic, the extent of electron scattering in the wall of a small hole has been studied in some detai?.

Coherent ele:... -agnetic effects have been ignored in the calculations summarized below. The transport calculation 
considered only point interactions of individual photons or eiectrons and specifically excluded collective effects which cause, or are the result of, external electric or magnetic fields or material conductivity. These effects which could reduce the beam radius, are thought to be less important in the solid beam dump than scattering and spitzer drag. (4) Nevertheless, bremsstrahlung and fluorescent photon generation were simulated in the calculation as well as secondary electrons due to auger, compton, pair production, and photo-electron processes.

\section{Conceptual Design for ATA Beam Dump}

Choice of material for a solid ATA beam dump is limited, first, by heat capacity and melting point during a single beam burst, and second, by thermal conductivity and geometrical configuration during continuous pulsing at the highest mean rate. Power density within the beam footprint is relatively insensitive to the choice of material since the electron range at this energy varies only in the range $15-25 \mathrm{gm} / \mathrm{cm}^{2}$ among the natural elements and the rate of energy deposition is approximately $1.5-2 \mathrm{MeV} / \mathrm{gm} / \mathrm{cm}^{2}$ due to collisions. Of course radiation is important in high $z$ materials but this energy deposition is spread over a large volume. Table I contains physical constants for several candidate materials. These data are used to roughly estimate the maximum allowed current density during a burst of ten ATA pulses (each $50 \mathrm{~ns}$ width) without melting the dump. Cooling during the burst is neglected. Clearly carbon or beryllium are favored choices since a11 others restrict the current density to values lower than that which occurs in the ATA beam at $r=1 \mathrm{~cm}$ radius. These two materials are also good choices (though not the lest) on the basis of thermal conductivity. 
High thermal conductivity is criticaliy important because the dump must be very thick $\left(>30 \mathrm{gm} / \mathrm{cm}^{2}\right)$ in order to stop the beam so that axial conduction does not provide significant cooling, as is the case in thin dumps used at low energy. Finally, photo-neutron production is important as far as radiation protection of nearby materials and diagnostic instruments. Since photoneutron yield from carbon is somewhat lower than from beryllium (5-8), the former material was chosen for these calculational studies. Although several mechanical arrangements of the dump are currently being studied, all would appear to the beam as approximately homogeneous cylinders of a single element.

\section{Solid Dump Power Density Profile}

Electron and photon energy deposition were calculated in the beam dump as follows. A beam of $50 \mathrm{MeV}$ electrons was assumed to enter a solid cylinder ( $R$ $=8 \mathrm{~cm})$ of carbon. The beam was parallel to the dump axis and entered unifarmly over a disc extending to $r=1 \mathrm{~cm}$. Current density was zero for $r>$ $1 \mathrm{~cm}$. Since conduction cooling is inadequate to remove the average beam power at $5 \mathrm{pps}^{(2)}$ the dump is expected to be segmented into many thin foils in arder to allow additional themal radiation cooling. This configuration is approximated in the calculation by extending the dump to a length of $L=100$ $\mathrm{cm}$ and reducing the carban density to $p=0.34 \mathrm{gm} / \mathrm{cm}^{3}$ so that the effective dump thickness is $34 \mathrm{gm} / \mathrm{cm}^{2}$, or about 1.3 mean ranges (5) at 50 MeV. 
Electron and gamma ray spectra were calculated at six planes located at $Z$ $=5,10,25,50,75$ and $100 \mathrm{~cm}$. Spectra within the annular bands defined by $r$ $=1,2,3,4,5,6,8 \mathrm{~cm}$ were used to determine the net electron and photon energy transported into, and out of, each annular zone defined by the above planes and radii. That energy was divided by the mass of the respective zone to determine the energy deposition density due to one $50 \mathrm{MeV}$ electron incident on the dump. In cases where statistics were poor the energy depositions in several radially concentric zones have been added and the result is an average over those zones. Fig. 1 sumarizes the results of the power density calculation. Two ordinates are given. The first (left) corresponds to the energy deposition density due to a single beam electron. The second ordinate (right) corresponds to the average power density when the mean beam current is $2.5 \mathrm{~mA}$ (5 pps and $50 \mathrm{~ns}$ pulse width). Data in Fig. 1 includes energy deposited by both photons and electrons in the carbon dump, and is averaged over the radij indicated by the width of the horizontal bars.

It is clear that the energy deposition profile spreads rapidly as the beam penetrates the dump. In the range $Z=10-25 \mathrm{~cm}$ the central power density is reduced by $1 / 3$ and in the range $z=25-50 \mathrm{~cm}$ the power density profile is expanded so that it is reasonably flat out to $r=4 \mathrm{~cm}$. Energy spectra of photons escaping the dump have also been calculated and results are shown in Fig. 2. The resulting spectrum is a characteristic brensstrahlung spectrum with minor fluctuations due to the limited statistics in the calculation. 


\section{Multiple Electron Scattering in Beam Dump Collimators}

The ATA beam dump is expected to provide an array of beamlets extracted through small $(r=.05 \mathrm{~cm})$ holes in the dump. Current measurements on these beamlets provides information on the beam profile as well as emittance. Angular divergence measurements also will be used to determine emittance. Finally, a bending magnet will be used for time resolved energy analysis. In all of these diagnostic measurements the importance of multiple scattering in the walls of the callimating hole must be assessed as it may affect the resulting data. Electron transport calculations have been carried out for several configurations in order to determine which scattering regions contribute the most to unwanted electron transport.

Three collimator designs were considered in these calculations. The first two consisted of a single hole bored through a carbon block $(p=2.25$ $\mathrm{gm} / \mathrm{cm}^{3}$ ) of thickness $15 \mathrm{~cm}$ (about 1.5 mean ranges). One had aperture radius $r=0.1 \mathrm{~cm}$, the other $r=1 \mathrm{~cm}$. In order to study the importance of scattering in the wall of the collimator, electrons were given initial trajectories which intersected the wall so that none would pass through without scattering. Angles of incidence were varied in the range $0.5-40$ mrad and initial entry points were in the ranges $Z=3-4 \mathrm{~cm}, Z=7-8 \mathrm{~cm}$, and $Z=12-13 \mathrm{~cm}$. A schematic representation of the problem geometry is shown in Fig. 3. Some calculations were continued until the electron energy was reduced below $0.5 \mathrm{MeV}$, at the expense of considerable computer time, while others terminated the electrons at $25 \mathrm{MeV}$ in order to reduce computational time requirements. 
Fig. 4 shows the energy spectra of electrons emerging from the back side of a beam dump with a $r=0.1 \mathrm{~cm}$ collimator. Fig $4(\mathrm{a})$ corresponds to illumination over the interval $z=3-4 \mathrm{~cm}$, Fig $4(b)$ to illumination over $z=$ $7-B \mathrm{~cm}$, and Fig. 4 (c) corresponds to $z=12-13 \mathrm{~cm}$ illumination. Of course the peak transmitted electron intensity increases as the area of illumination is nearer the exit $(z=12-13 \mathrm{~cm})$ and also as the initial angle of incidence decreases. The important feature to note is that the scattered electron intensity at high energies is generally small. The fraction of incident electrons which lose less than $1 \mathrm{MeV}$ in scattering along the hole varies from $.003-.023$ for electrons incident at $Z=3-4 \mathrm{~cm}$, to .007-.28 for electrons incident at $z=12-13 \mathrm{~cm}$. The fraction of electrons losing less than $5 \mathrm{MeV}$ is only slightly larger. Some of the electron intensity emitted from the back of the dump in this calculation is due to compton scattering of bremsstrahlung radiation but most is due to electrons which have lost energy scattering in carbon. Thus the spectrum of electrons at the back rises at low energy due to the presence of compton electrons and a peak due to transmitted primary electrons is observed whose energy decreases as the electrons traverse a greater thickness of solid material; i.e. a peak is observed at $\sim 40$ MeV for $z$ $=12-13 \mathrm{~cm}$ illumination while it occurs at $\sim 20$ MeV for $z=7-8 \mathrm{~cm}$ illumination.

The results of the above calculations may be used to assess an experimental diagnostic by comparing the intensity of scattered electrons above a hypothetical energy threshold to that of the unscattered electrons, i.e. those with initial trajectories extending through the exit aperture. The latter depends on the angular distribution of the entrant beam (emittance) and on angular bias. 
Calculations have been carried out for larger holes with $r=1 \mathrm{~cm}$, and the results are sumarized in Fig. 5. Transmission of scattered electrons is greater with a larger exit aperture due to the larger solid angle for lossless exit. Otherwise the results are qualitatively similar to those for the smaller diameter collimator .

Finally, a calculation has been carried out for a $r=.05 \mathrm{~cm}$ diam $\times 100 \mathrm{~cm}$ long hois in law density carbon $\left(\rho=0.34 \mathrm{gm} / \mathrm{cm}^{3}\right)$. Fig. 6 summarizes the results. In this case the electron source is assumed to illuminate the entire length of the wall from a point on the collimator axis at $Z=-10 \mathrm{~cm}$. An angular distribution was assumed which generated electrons uniformly over the spherical surface between the maximum cone angle $\theta=5$ mrad and the minimum cone angle defined by the trajectory to a point on the wall at $Z=99 \mathrm{~cm}$. Thus, almost the entire wall is illuminated by electrons at initial incidence angles less than 5 mrad.

It is clear from the figure that about $10^{-4}$ of the electrons are transmitice with less than $1 \mathrm{MeV}$ energy loss and only about $10^{-3}$ are transmitted with less than $5 \mathrm{MeV}$ energy loss. A beam with nominal angular divergence of $5 \mathrm{mrad}$ would experience an attenuation of unscattered electrons on the order of $10^{-2}$ in transiting this collimator. In this case the signal to background (ratio of unscattered electrons to number scattered electrons with less than $1 \mathrm{MeV}$ energy loss) is of order 100 but decreases to 10 if the background includes electrons which have lost up to $5 \mathrm{MeV}$. 
Finally, the electron background due to electrons transmitted through a solid carbon dump has been calculated for a dump of thickness $L=34$ $\mathrm{gm} / \mathrm{cn}^{2}$. Fig. 7 shows the resulting electron spectrum. It is clear from the figure that straggling and compton scattering produce some eiectrons at the back face of the dump. For a dump such as the one considered where the thickness is about 1.3 mean ranges, the electron intensity above $40 \mathrm{MeV}$ is about $10^{-5}$ of the incident intensity. However, the total electron penetration is on the order of a few percent.

\section{Conclusions}

It was shown previously that very few solids will withstand the power density in the ATA beam at $1 \mathrm{~cm}$ radius without melting during a burst of ten pulses. None have sufficient thermal conductivity to dissipate the continuous thermal load at 5 pps by thermal conduction alone. Only carbon and beryllium will withstand a beam burst and both require supplementary rapid cooling by thermal radiation or by wisplacement of the heated volume between bursts. Carbon has been studied as a candidate material due to its lower photoneutron generation in the ATA beam.

Monte-carlo electron-photon transport calculations show that the scattering in the dump reduces the central current (and power) density substantially at a penetration of 0.15 mean electron ranges so that thermal loading is primarily important in a relatively small volume at the entrant face of the dump. At larger penetrations the power density due to beam and secondary energy deposition is nearly flat to large radii. The results on which this conclusion is based did not include coherent interactions which might focus the beam, but a rough calculation for a conducting foil indicates only a small focussing effect due to elimination of the electric field. 
Calculations of the scattered electron background near a collimator hole in the dump show that, under rezsonable conditions of a diagnostic measurement, the scattered intensity is less than the unscattered intensity by one to two decades. This remains true even when secondary electrons are included. Thus the interference of scattered or secondary electrons with emittance, profile, or energy measurements should be relatively unimportant so long as the experiment is able to distinguish electrons which have lost more than $5 \mathrm{MeV}$ from those of interest. 


\section{Physical Properties of Candidate ATA Dump Materials}

\begin{tabular}{|c|c|c|c|c|c|c|c|}
\hline Element & $\begin{array}{l}\mathrm{Cp} \\
\text { at } 1500^{\circ} \mathrm{C} \\
\left(\mathrm{J} / \mathrm{gm}^{\circ} \mathrm{K}\right)\end{array}$ & $T_{\text {fusion }}-300^{\circ} \mathrm{k}$ & $\begin{array}{l}k \\
\text { at } 1500^{\circ} \mathrm{C} \\
\left(\frac{\text { watt }}{c m^{2} \cdot k}\right)\end{array}$ & $\begin{array}{l}\left.\frac{d E}{d X}\right|_{\text {collijions }} \\
\left(\frac{\mathrm{MeV} \mathrm{\textrm {cm } ^ { 2 }}}{g m}\right)^{\text {m }}\end{array}$ & $\begin{array}{l}\text { Range } \\
\text { O } 50 \mathrm{MeV} \\
\text { (cm) }\end{array}$ & $\begin{array}{l}\sigma(r, n) \\
(\mathrm{mb})\end{array}$ & $\begin{array}{l}\text { Maximum } \\
\text { Current } \\
\text { Density } \\
\left(\frac{\text { Amp }}{\mathrm{cm}^{2}}\right)\end{array}$ \\
\hline $\mathrm{Be}$ & 3.03 & 1260 & 0.8 & 1.69 & 14 & 2.5 & 4500 \\
\hline C & 1.98 & 3800 & $0.2-17.0$ & 1.90 & 10 & 2.1 & 7900 \\
\hline A1 & 1.18 & 633 & 2.0 & 1.79 & 7.7 & 7.2 & 840 \\
\hline$T i$ & 0.69 & 1643 & 0.25 & 1.7 & & & 1300 \\
\hline$v$ & 0.72 & 1875 & 0.35 & 1.7 & & 31 & 1600 \\
\hline $\mathrm{Fe}$ & 0.66 & 1509 & 0.72 & 1.66 & 2.3 & & 1200 \\
\hline $\mathrm{Cu}$ & 0.49 & 1057 & 3.2 & 1.62 & 2.0 & 34 & 640 \\
\hline $\mathrm{Ni}$ & 0.62 & 1426 & 0.8 & 1.62 & & 19 & 1100 \\
\hline Mn & 0.84 & 1217 & .08 & 1.66 & & 39 & 1200 \\
\hline $\mathrm{Zr}$ & 0.34 & 1825 & .22 & 1.5 & & 65 & 830 \\
\hline Mo & 0.33 & 2590 & 1.0 & 1.5 & & 90 & 1100 \\
\hline Ta & 0.16 & 1950 & 0.62 & 1.38 & & 205 & 450 \\
\hline$W$ & 0.16 & 3353 & 1.06 & 1.38 & 0.76 & 203 & 730 \\
\hline U & 0.20 & 1105 & 0.5 & 1.32 & 0.74 & 290 & 330 \\
\hline
\end{tabular}

*Assuming material is allowed to reach its melting point following rapid delivery of ten-50 ns pulses. 
REFERENCES

1. H.A. Barletta, "Generating Intense Electron Beams for Military Applications," LLNL, Energy and Technology Review, December, 1981

2. Denn is Slaughter, "Constraints on the ATA Beam Dump Design", LLNL, ATA Note 178, March 1982.

3. H.P. Colbert, "SANDYL: A Computer Program for Calculating Combined Photon-Electron Transport in Complex Systems", Sandia Laboratories, Livermore, SLL-74-0012 (1974).

4. A.C. Paul, private communication

5. M.J. Berger, S.M. Seltzer, Tables of Energy Losses and Ranges of Electrons and Positrons, Nat. Aeronautics and Space Admin, NASA-SP-3012 (1964).

6. Nuclear Data Tables $\underline{A}$, Vol. 2, (1966).

7. W.C. Barber, W.D. George, Phys. Rev 116, 1551 (1959).

8. W.P. Swanson, Radiological Safety Aspects of the Operation of Electron Linear Accelerators, IAEA Tech Report Series No. 188 (1979). 
Figure captions:

1. Energy (power) density in a carbon beam dump due to electron energy deposition. Ordinate gives differential energy deposited (MeV/gm) per incident electron for a 50 MeV beam with radius $1 \mathrm{~cm}$.

2. Energy spectrum of photons escaping from the ATA beam dump. Data are normalized to one primary electron: a) Rear face of the dump, b) Cylindrical (side) face.

3. Problem geometry for collimator scattering calculation. Simulation assumes electrons enter wall of collimator between $z_{1}$ and $z_{2}$ at incident angles between $\theta$ min and $\theta$ max.

4. Energy spectra of scattered electrons at the exit face of an ATA collimator with $r=0.1 \mathrm{~cm}$, length $15 \mathrm{~cm}$ in $=2.25 \mathrm{gm} / \mathrm{cm}^{2}$ carbon. Data are normalized to one bean electron. The plotting characters refer to the initial angle of incidence. Mean incidence angles considered Were: 1) $0.5 \mathrm{mrad}$, 2) $1.0 \mathrm{mrad}, 3) 3.0 \mathrm{mrad}$, 4) $10 \mathrm{mrad}$, 5) $40 \mathrm{mrad}$, and 6) $100 \mathrm{mrad}$.

a) wall illuminated over $Z=3-4 \mathrm{~cm}$

b) wall illuminated over $Z=7-8 \mathrm{~cm}$

c) wall illuminated over $z=12-13 \mathrm{~cm}$

5. Energy spectra of scattered electrons at the exit face of an ATA collimator with radius $r=1 \mathrm{~cm}, L=15 \mathrm{~cm}$ in $\rho=2.25 \mathrm{gm} / \mathrm{cm}^{3}$ carbon. Data normalized to one beam electron and plotting characters are the same as in Fig. 4.

a) wall illuminated over $z=3-4 \mathrm{~cm}$

b) wall illuminated over $z=7-8 \mathrm{~cm}$

c) wall illuminated over $Z=12-13 \mathrm{~cm}$

6. Energy spectra of scattered electrons at the exit face of an ATA collimator with radius $r=.05 \mathrm{~cm}, L=100 \mathrm{~cm}$ in $\rho=0.34 \mathrm{gm} / \mathrm{cm}^{3}$ carbon. Source illuminates entire wall from a point on axis at $Z=-10 \mathrm{~cm}$.

7. Energy spectra of electrons transmitted through a solid carbon beam dump, $\mathrm{L}=34 \mathrm{gm} / \mathrm{cm}^{2}$. 


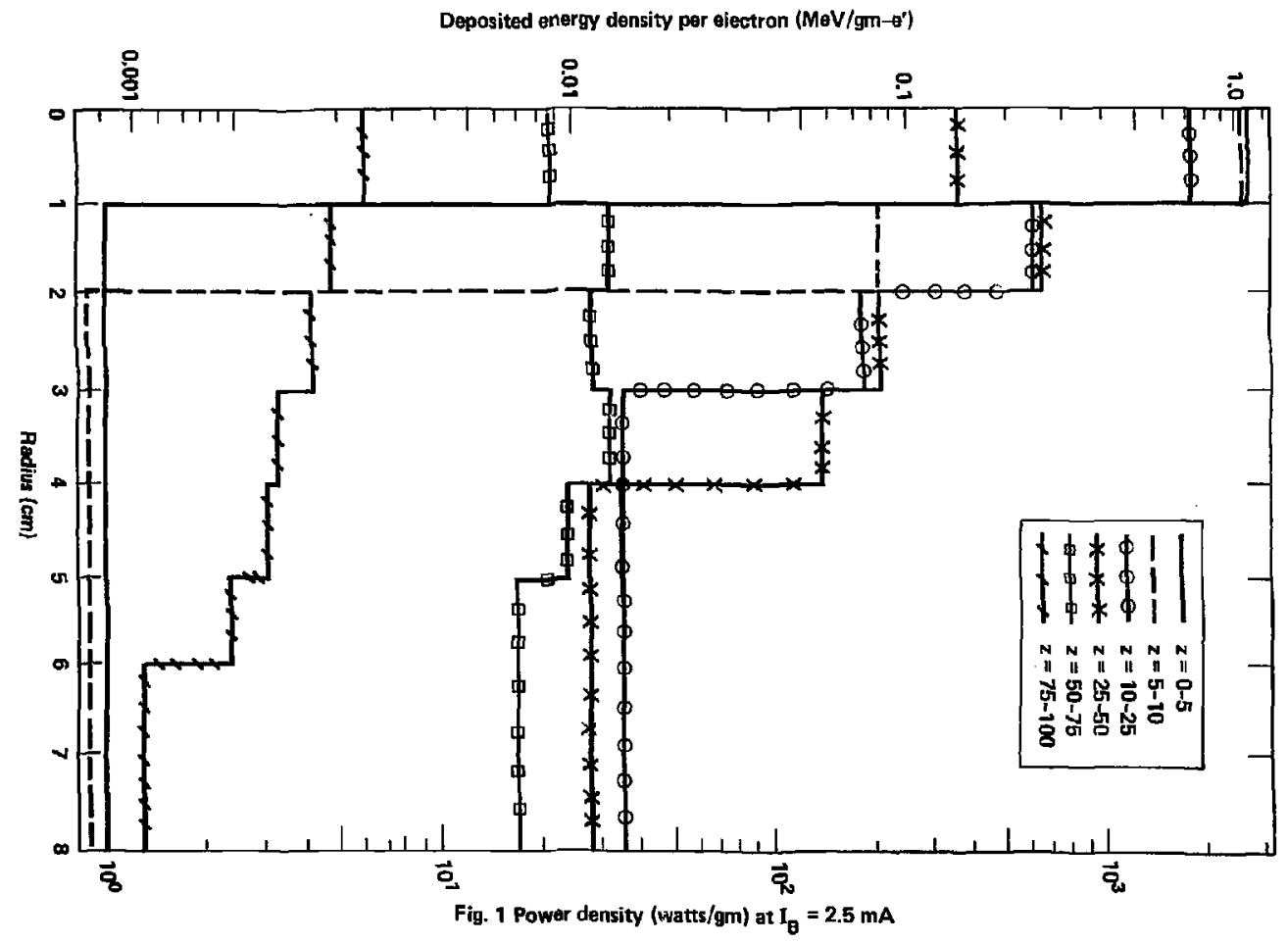




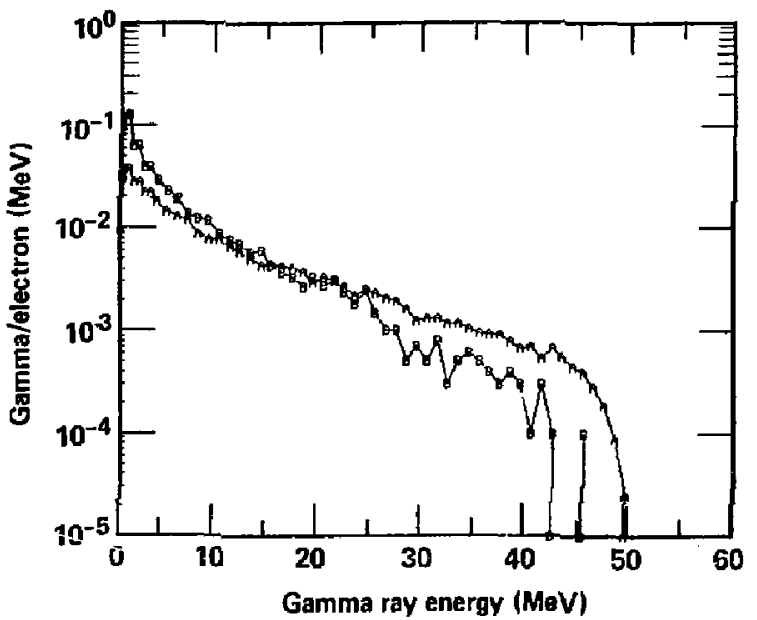




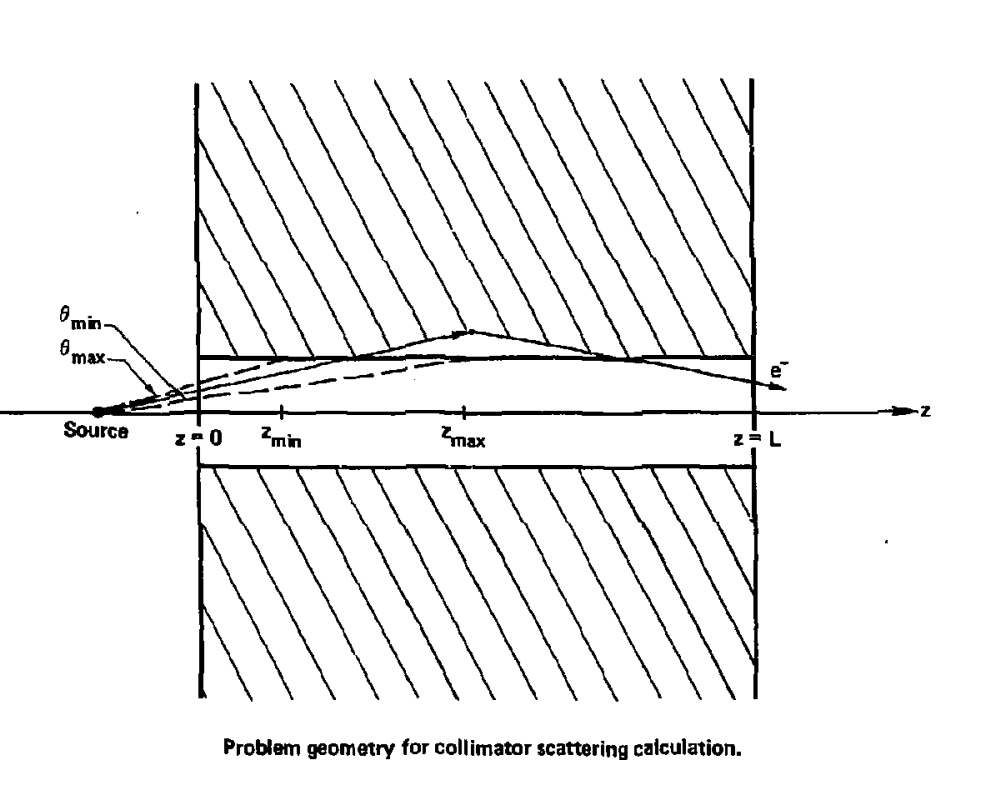




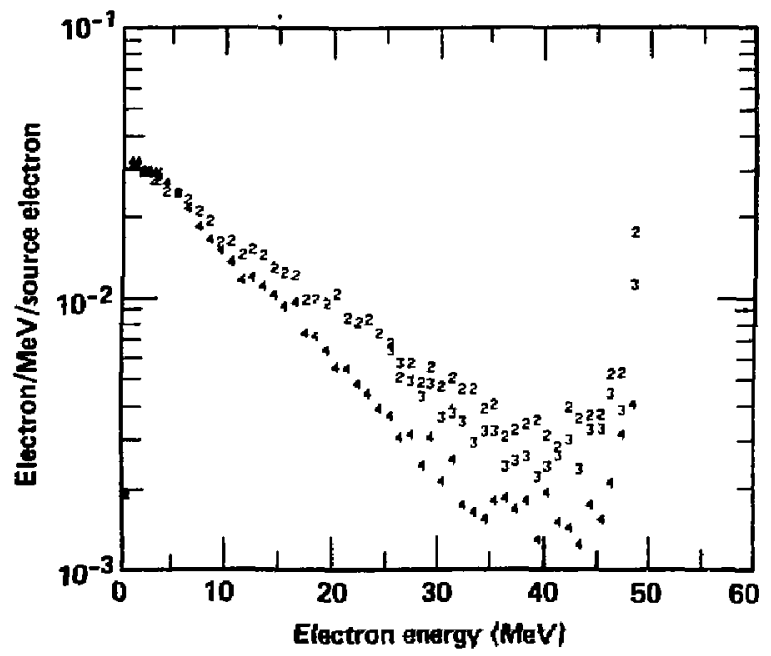




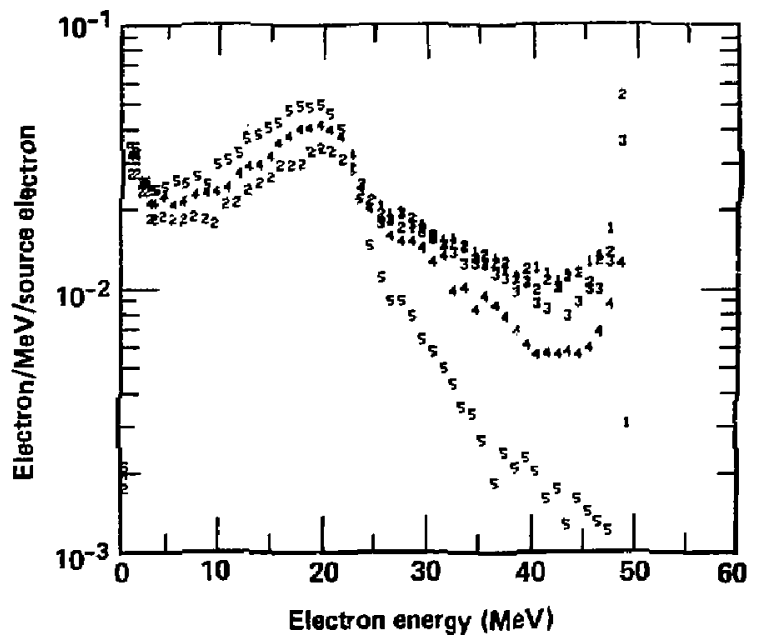




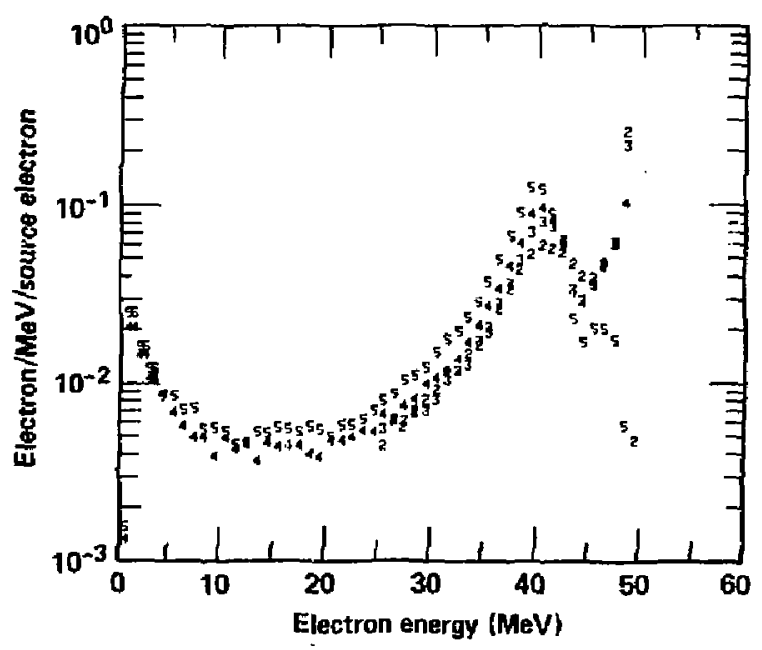




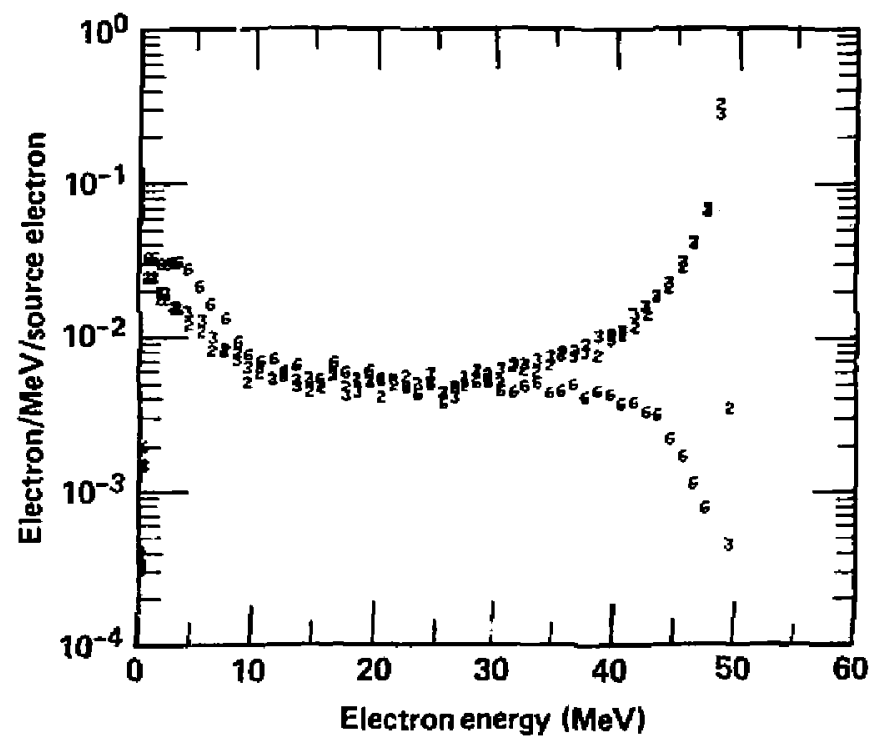




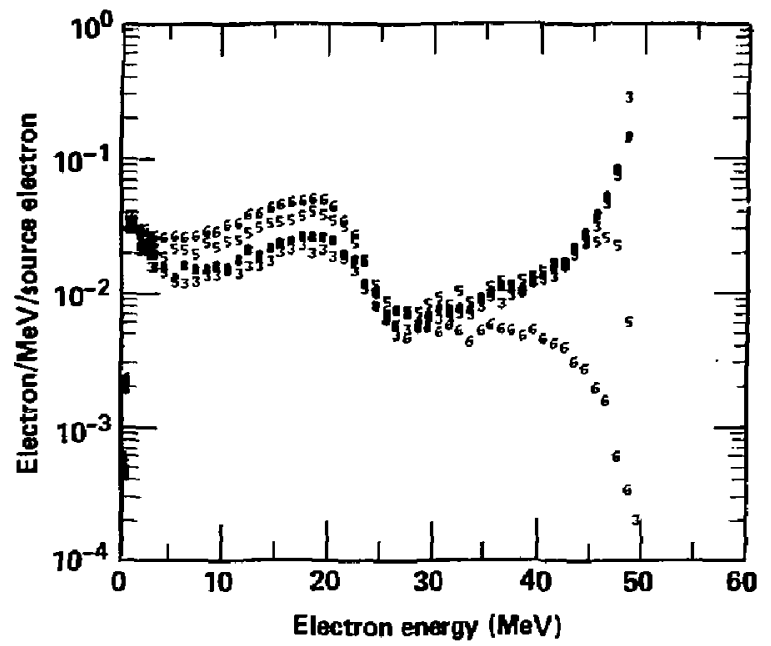




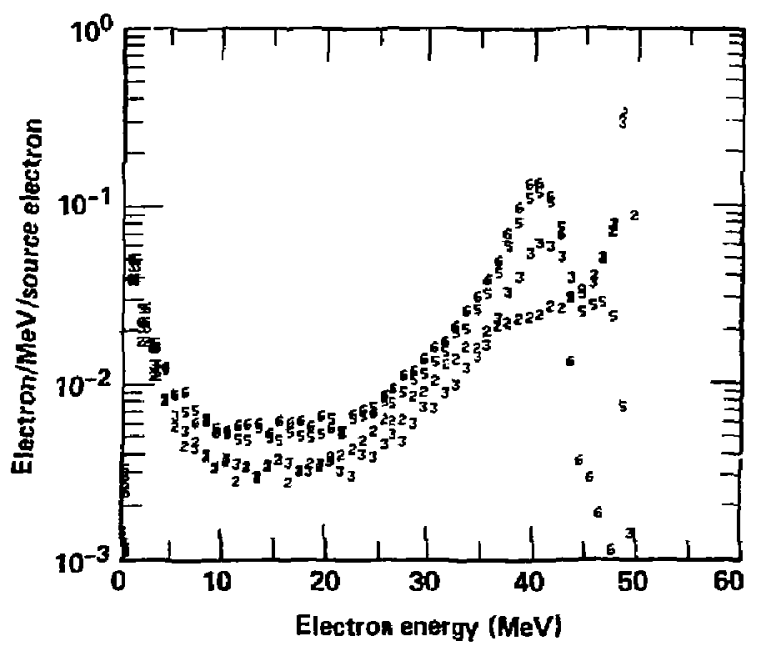




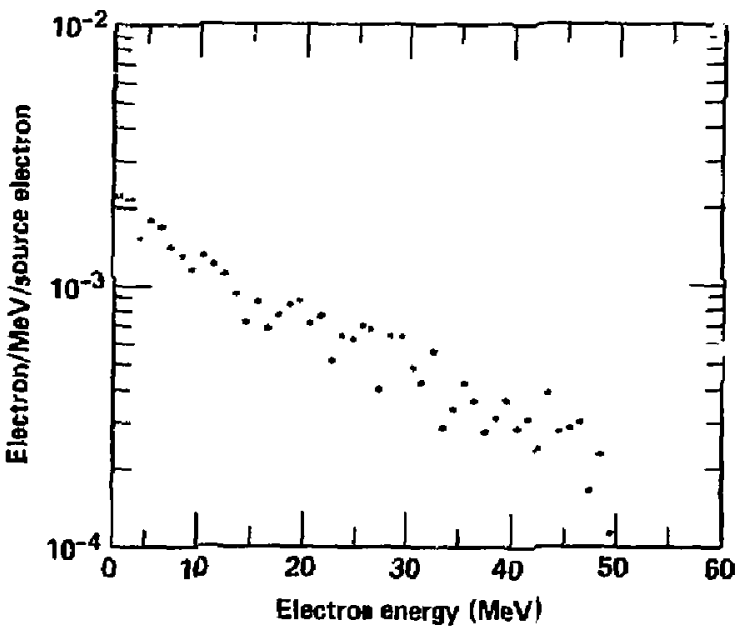




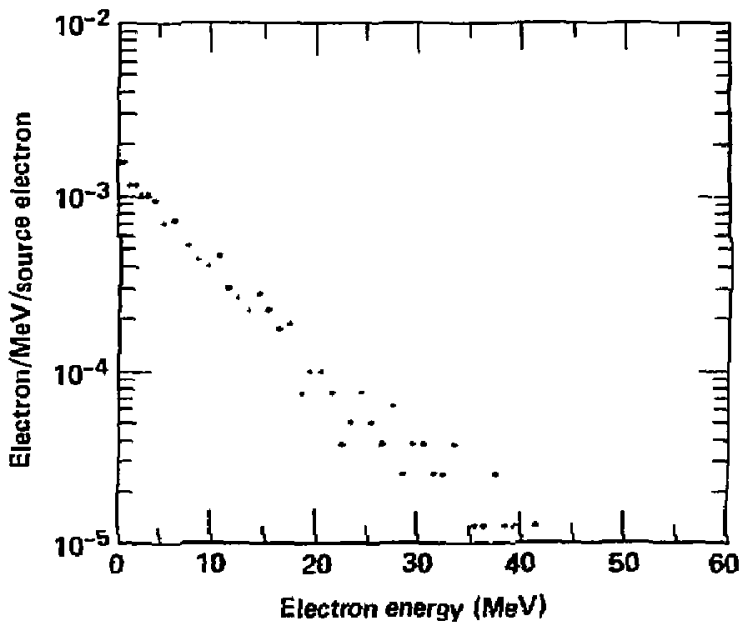

